九州大学学術情報リポジトリ

Kyushu University Institutional Repository

\title{
Global time evolution of viscous vortex rings
}

Fukumoto, Yasuhide

Faculty of Mathematics, Kyushu University

http://hdl. hand le. net/2324/13203

出版情報 : MI Preprint Series. 2009-1，2009-01-16. 九州大学大学院数理学研究院 バージョン：

権利関係 : 


\section{Preprint Series}

Kyushu University

The Grobal COE Program

Math-for-Industry Education \& Research Hub

\section{Global time evolution of viscous vortex rings}

\section{Y. Fukumoto}

\section{2009-1}

( Received January 16, 2009 )

Faculty of Mathematics

Kyushu University

Fukuoka, JAPAN 


\title{
Y. Fukumoto
}

\section{Global time evolution of viscous vortex rings}

Received: date / Accepted: date

\begin{abstract}
This article gives an overview of growing knowledge of translation speed of an axisymmetric vortex ring, with focus on the influence of viscosity. Helmholtz-Lamb's method provides a short-cut to manipulate the translation speed at both small and large Reynolds numbers, for a vortex ring starting from an infinitely thin core. The resulting asymptotics significantly improve Saffman's formula (1970) and give closer lower and upper bounds on translation speed in an early stage. At large Reynolds numbers, Kelvin-Benjamin's kinematic variational principle achieves a further simplification. At small Reynolds numbers, the whole life of a vortex ring is available from the vorticity obeying the Stokes equations, which is closely fitted, over a long time, by Saffman's second formula.
\end{abstract}

Keywords vortex ring $\cdot$ Helmholtz-Lamb's method $\cdot$ variational principle $\cdot$ viscous decay

PACS 47.32.cf · 47.15.ki $\cdot$ 47.15.G-

\section{Introduction}

Vortex rings are ubiquitous coherent structures in high-Reynolds-number flows, and are of fundamental importance in fluid mechanics as indicated by the fact that visualized cross-section of a vortex ring is put on the cover of Batchelor's textbook [1] ${ }^{1}$. Okabe [2] recollected that such a beautiful pattern was gained only once or twice among a hundred trials, and an interval of 20 or 30 minutes between trials were required to wait for the water in a tank becoming clean and still. Vortex rings are used for producing thrust and lift by insects, fishes and animals. Vortex rings are capable of transporting neutrally buoyant materials. Recently they find their utility for creating virtual reality in the field of entertainment. There is an attempt to use an air cannon, as a means of olfactory display, to deliver smells encapsulated in a vortex ring to a targeted person. In a theater, virtual reality contents are created solely by image and sound. Reality is enhanced if we appeal to tactile display. A mini-theater is planned in which air cannons are designed to produce vortex rings, in synchronization with the image and the sound, so that the audience experiences direct impact and freshness [3].

These applications to entertainment necessitate controlled vortex rings, and raise questions pertaining to an inverse problem. When does a vortex ring arrive at a specified point? How far does the ring travel? How large the vortex ring has grown at the moment of impact? The purpose of this article is to give a possible answer to these questions, under restricted situations, while giving a brief survey of the growth of knowledge of traveling speed of a vortex ring.

Y. Fukumoto

Graduate School of Mathematics, Kyushu University 33, Fukuoka 812-8581, Japan

Tel.: +81-92-642-2762

Fax: +81-92-642-2779

E-mail: yasuhide@math.kyushu-u.ac.jp

1 G. K. Batchelor knew Okabe-Inoue's photographs through the annual report of their Institute (private communication with J. Okabe). 
Study on motion of vortex rings started simultaneously with the birth of the field of vortex dynamics when Helmholtz introduced the vorticity and proved its property of being frozen into the fluid in his seminal paper a century and a half ago [4]. By an elaboration from the Euler equations, now being widely known through Lamb's textbook [5]. Helmholtz had reached an identity for $U$ of a thin axisymmetric vortex ring, steadily translating in an inviscid incompressible fluid of infinite extent. Helmholtz-Lamb's method is expounded in Sect. 3. Ignoring constant terms compared with a logarithmically large term, Helmholtz related the translation speed $U$ to the total kinetic energy $H$ and the hydrodynamic impulse $P_{z}$, and made a crude estimation of this relation for a thin core, of core radius $\sigma$ and ring radius $R_{0}$ carrying the circulation $\Gamma$, as

$U \approx H / P_{z} \approx \frac{\Gamma}{4 \pi R_{0}}\left[\log \left(\frac{8 R_{0}}{\sigma}\right)+\right.$ const. $]$.

Continuing Helmholtz's analysis, Kelvin (1867) determined the constant to be $-1 / 4$ in the above formula, for a distribution of vorticity, in the core, proportional to the distance from the axis of symmetry. Only the resulting expression, without derivation, was recorded in an appendix to Tait's English translation of Helmholtz's paper [4].

On those days, vortex rings were hot as possible entities of atoms embedded in the ether. The implication of Helmholtz' laws, invariance in time of the circulation and linkages of vortex lines, led Kelvin to this belief. J. J. Thomson pursued the idea of the vortex atoms [6]. To derive the translation speed, he employed a straightforward approach of taking the boundary of the core as a free boundary coincident with a streamline, but Kelvin's formula was unattained; the constant that he gave was -1 rather than -1/4. This discrepancy was traced back to his insufficient treatment of the Biot-Savart law for deriving the velocity field around the vortex core, and was rescued by Hicks [7]. An interpretation of this difference was explained in Sect. 2.

By adapting his technique for calculating the gravity potential around the Saturn ring, Dyson [8] contrived an ingenious systematic perturbation method evaluating the Biot-Savart law, and thereby overcame the difficulty to proceed to third (virtually fourth) order in $\varepsilon=\sigma / R_{0}$.

$U=\frac{\Gamma}{4 \pi R_{0}}\left\{\log \left(\frac{8 R_{0}}{\sigma}\right)-\frac{1}{4}-\frac{3 \sigma^{2}}{8 R_{0}^{2}}\left[\log \left(\frac{8 R_{0}}{\sigma}\right)-\frac{5}{4}\right]+O\left(\varepsilon^{4} \log \varepsilon\right)\right\}$.

The same result was reached, in a thin limit, by transforming the free boundary-value problem of the Euler equations into an integral equation $[9,10]$. This integral equation was solved for the whole family of axisymmetric vortex rings with vorticity in the core being proportional to the distance from the symmetric axis. This is referred to as Fraenkel-Norbury's family [11]. Fraenkel [10] pointed out that, by a suitable renormalization of thickness parameter $\varepsilon$ with which the fat limit corresponds to $\varepsilon=\sqrt{2}$, (2) is applicable, with an error no more than 5 per cent, to the translation speed of Hill's vortex, the fat limit $(\varepsilon=\sqrt{2})$. This agreement has inspired us to generalize Dyson's formula to more realistic vortex rings $[12-14]$.

To $O(\varepsilon)$, Kelvin's formula was extended to allow for an arbitrary distribution of vorticity as

$U_{0}=\frac{\Gamma}{4 \pi R_{0}}\left\{\log \left(\frac{8 R_{0}}{\sigma}\right)+A-\frac{1}{2}+O(\varepsilon, \varepsilon \log \varepsilon)\right\} ; \quad A=\lim _{r \rightarrow \infty}\left\{\frac{4 \pi^{2}}{\Gamma^{2}} \int_{0}^{r} r^{\prime} v_{0}\left(r^{\prime}\right)^{2} \mathrm{~d} r^{\prime}-\log \left(\frac{r}{\sigma}\right)\right\}$,

where $v_{0}(r)$ is the local velocity of circulatory motion of the fluid, in the cross-section, around the toroidal center circle, as a function only of the local distance $r$ from the circle $[9,16,17]$. The functional form of $v_{0}(r)$ remains indeterminate, but, if the viscosity $\nu$ is called into play, a unique profile as a function of time $t$ is singled out once the initial profile is given. The small parameter gives way to $\varepsilon=\sqrt{\nu / \Gamma}[12,15]$. Suppose that, at time $t=0$, the vorticity is concentrated on a circle of radius $R_{0}$, the leading-order terms of toroidal vorticity $\zeta_{0}$ and azimuthal velocity $v_{0}$ are provided by the Oseen diffusing vortex

$\zeta_{0}=\frac{\Gamma}{4 \pi \nu t} e^{-r^{2} / 4 \nu t}, \quad v_{0}=-\frac{\Gamma}{2 \pi r}\left(1-e^{-r^{2} / 4 \nu t}\right)$.

The minus sign in $v_{0}$ comes from our choice of local azimuthal coordinates (see Fig. 1) Saffman [16] showed that viscous diffusion of vorticity gets along with Helmholtz-Lamb's identity and obtained the translation speed of a vortex ring in a viscous fluid, simply by inserting (4) into (3), as

$U_{0}=\frac{\Gamma}{4 \pi R_{0}}\left\{\log \left(\frac{8 R_{0}}{2 \sqrt{\nu t}}\right)-\frac{1}{2}(1-\gamma+\log 2)+O\left[\frac{\nu t}{R_{0}^{2}}, \frac{\nu t}{R_{0}^{2}} \log \left(\frac{\nu t}{R_{0}^{2}}\right)\right]\right\}$, 
where $\gamma=0.57721566 \cdots$ is Euler's constant ${ }^{2}$. The radius of viscous core is $\sigma \approx 2 \sqrt{\nu t}$, and (5) is valid at early times when the core is thin $\sqrt{\nu t} \ll R_{0}$. The same formula was derived via the method of matched asymptotic expansions [15,17]. Recently, Fraenkel-Saffman's formula (3) is extended to $O\left(\varepsilon^{3}\right)$. In other words, Dyson's formula (2) is generalized to accommodate a general distribution of vorticity. At the same time, an extension of Saffman's formula to $O\left(\varepsilon^{3}\right)$ is achieved. A brief announcement of these results is given in ref. [14]. It is worth emphasizing that Helmholtz-Lamb's method is far more efficient than matched asymptotic expansions. The former leads us to the correction of $O\left(\varepsilon^{3}\right)$ to translation speed of a vortex ring without having to enter into the $O\left(\varepsilon^{3}\right)$ velocity field.

The development of theories of vortex rings attained before the early 90 s is well recorded in refs. [18-21]. This article supplements these by focusing on theoretical development made after that, with particular emphasis put on higher-order extension of velocity formula and on viscous vortex rings at both very high and very low Reynolds numbers. Dyson's technique for asymptotic development of the Biot-Savart law is instrumental for deriving the expression of the velocity field near the core. Before going to a description of higher-order extension of the translation speed, we sketch the essence of this technique in Sect. 2. Thereafter, Sect. 3 gives an account of Helmholtz-Lamb's method, and, resorting to this method, presents the third-order correction to translation speed in Sect. 4.

A variational principle brings a further simplification in derivation for an inviscid vortex $[18,22$, 23]. Take the density of fluid to be $\rho_{\mathrm{f}}=1$ and define the hydrodynamic impulse by

$\boldsymbol{P}=\frac{1}{2} \iiint \boldsymbol{x} \times \boldsymbol{\omega} \mathrm{d} V$.

The translation velocity $\boldsymbol{U}$ of a vortex ring is then calculable through the variation

$\delta H-\boldsymbol{U} \cdot \delta \boldsymbol{P}=0$,

under the constraint that, for any smooth Lagrangian displacement of fluid particles, the vorticity is frozen into the fluid. Section 5 touches upon this principle, which is the theme of ref. [14]. We may view (7) as a refinement of the crude estimate (1). Behind (7) lies Kelvin's variational principle [24, $25]$, as generalized to make allowance for motion [26,27], that a stationary configuration of vorticity in an inviscid incompressible fluid, in a steadily moving frame, is realizable as an extremal of energy on an iso-vortical sheet. Intriguingly, the same principle encompasses motion of a vortex ring ruled by the cubic nonlinear Schrödinger equation, which serves as a model for superfluid liquid helium and a Bose-Einstein condensate, at zero temperature [29].

The rest of paper is concerned with motion of a vortex ring at very low Reynolds numbers. There is no permanent vortex ring. Without unstable waves, a vortex ring dies away due to the action of viscosity while entraining surrounding irrotational flows [30-32]. The decaying laws of an axisymmetric vortex rings in a viscous fluid were handled separately in the literature. Recently a solution of an initialvalue problem valid over the whole time range is found for an axisymmetric vortex ring at low Reynolds numbers $[33,34]$ which enables us to view, in perspective, the early-time behavior (5), Saffman's second law valid in the matured stage $\sqrt{\nu t} \approx R_{0}[16]$, and the decaying law

$U=\frac{7 P_{z}}{240 \sqrt{2}(\pi \nu t)^{3 / 2}} \approx 0.0037037954 \frac{P_{z}}{(\nu t)^{3 / 2}}$,

at large times $\sqrt{\nu t} \gg R_{0}[20,35-37]$. A concise description of the low-Reynolds-number solution is given in Sect. 6. The last section (Sect. 7) is devoted to a summary and conclusions.

\section{Asympytotic development of Biot-Savart law: Dyson's technique}

Dyson's ingenious technique [8] is, in effect, indispensable for manipulating asymptotic expansions of the flow field around the vortical core to high orders. Here we delineate its essence as generalized to an arbitrary distribution, including a continuous one, of vorticity [12].

Consider an axisymmetric vortex ring of circulation $\Gamma$ moving in an infinite expanse of fluid. Choose cylindrical coordinates $(\rho, \phi, z)$ with the $z$-axis along the axis of symmetry and $\phi$ along the vortex lines as shown in Fig. 1. We consider an axisymmetric distribution of vorticity $\boldsymbol{\omega}=\zeta(\rho, z) \boldsymbol{e}_{\phi}$ localized

\footnotetext{
${ }^{2}$ In (3) and (5), Saffman's estimate of the remaining terms has been improved [12].
} 


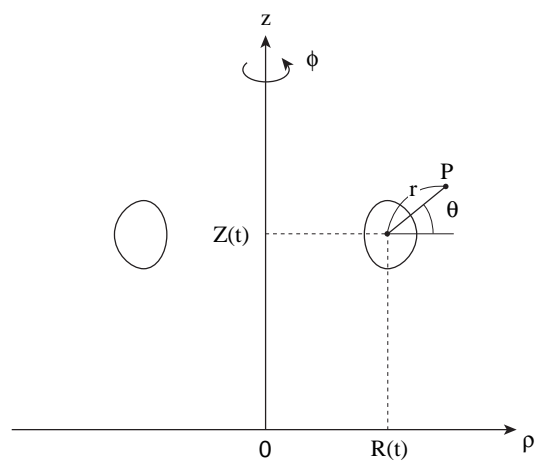

Fig. 1 Cylindrical and local moving coordinates

about the circle $(\rho, z)=(R(t), Z(t))$, where $\boldsymbol{e}_{\phi}$ is the unit vector in the azimuthal direction. The vector potential $\boldsymbol{A}(\boldsymbol{x})$ of the velocity field $\boldsymbol{u}(\boldsymbol{x})(\boldsymbol{u}=\nabla \times \boldsymbol{A})$ has azimuthal component only. We introduce the Stokes streamfunction $\psi$ by $\boldsymbol{A}(\boldsymbol{x})=-(\psi / \rho) \boldsymbol{e}_{\phi}$. The requirement of vanishing the vector potential at infinity, that is $|\boldsymbol{A}| \propto 1 /|\boldsymbol{x}|^{2}$ as $|\boldsymbol{x}| \rightarrow \infty$, facilitates the calculation of the total kinetic energy. With this requirement, the Biot-Savart law is represented for the Stokes streamfunction as

$\psi(\rho, z)=-\frac{\rho}{4 \pi} \int_{-\infty}^{\infty} \int_{0}^{2 \pi} \int_{0}^{\infty} \frac{\zeta\left(\rho^{\prime}, z^{\prime}\right) \rho^{\prime} \cos \phi^{\prime} \mathrm{d} \rho^{\prime} \mathrm{d} \phi^{\prime} \mathrm{d} z^{\prime}}{\sqrt{\rho^{2}-2 \rho \rho^{\prime} \cos \phi^{\prime}+\rho^{\prime 2}+\left(z-z^{\prime}\right)^{2}}}$.

We introduce, in the meridional plane, local Cartesian coordinates $(\hat{x}, \hat{z})=(\rho-R, z-Z)$ centered at $(R(t), Z(t))$. Supposing a rapid decay of $\zeta(\boldsymbol{x})$ with the distance from the circle, we perform an asymptotic expansion of (9) valid near the core. The first of the key steps of Dyson's technique is to utilize the shift operator to rewrite (9) as

$\psi=-\frac{\rho}{4 \pi} \iint_{-\infty}^{\infty} \mathrm{d} \hat{x}^{\prime} \mathrm{dd} \hat{z}^{\prime} \zeta\left(\hat{x}^{\prime}, \hat{z}^{\prime}\right) \exp \left(\hat{x}^{\prime} \frac{\partial}{\partial R}-\hat{z}^{\prime} \frac{\partial}{\partial \hat{z}}\right) \int_{0}^{2 \pi} \frac{R \cos \phi^{\prime} \mathrm{d} \phi^{\prime}}{\sqrt{\rho^{2}-2 \rho R \cos \phi^{\prime}+R^{2}+\hat{z}^{2}}}$.

The asymptotic form of (9) is automatically generated by expanding the exponential function of the operators as

$$
\begin{aligned}
\psi(\rho, z)= & \iint_{-\infty}^{\infty} \mathrm{d} \hat{x}^{\prime} \mathrm{d} \hat{z}^{\prime} \zeta\left(\hat{x}^{\prime}, \hat{z}^{\prime}\right)\left\{1+\left(\hat{x}^{\prime} \frac{\partial}{\partial R}-\hat{z}^{\prime} \frac{\partial}{\partial z}\right)+\frac{1}{2 !}\left(\hat{x}^{\prime} \frac{\partial}{\partial R}-\hat{z}^{\prime} \frac{\partial}{\partial z}\right)^{2}\right. \\
& \left.+\frac{1}{3 !}\left(\hat{x}^{\prime} \frac{\partial}{\partial R}-\hat{z}^{\prime} \frac{\partial}{\partial z}\right)^{3}+\frac{1}{4 !}\left(\hat{x}^{\prime} \frac{\partial}{\partial R}-\hat{z}^{\prime} \frac{\partial}{\partial z}\right)^{4}+\cdots\right\} \psi_{\mathrm{m}}(\rho, z ; R) .
\end{aligned}
$$

Here

$\psi_{\mathrm{m}}(\rho, z ; R)=-\frac{\rho R}{4 \pi} \int_{0}^{2 \pi} \frac{\cos \phi^{\prime} \mathrm{d} \phi^{\prime}}{\sqrt{\rho^{2}-2 \rho R \cos \phi^{\prime}+R^{2}+(z-Z)^{2}}}$,

is the streamfunction for the flow induced by a circular line vortex of unit strength placed at $(R, Z)$, or a delta-function core $\zeta(\rho, z)=\delta(\rho-R) \delta(z-Z)$.

Observe that the monopole field (12) is symmetric with respect to interchange between $\rho$ and $R$. It follows from the connection between $\psi$ and $\zeta$ that, except at the core $(\rho, z)=(R, Z), \psi_{\mathrm{m}}$ obeys

$$
\left(\frac{\partial^{2}}{\partial R^{2}}+\frac{\partial^{2}}{\partial z^{2}}\right) \psi_{\mathrm{m}}=\frac{1}{R} \frac{\partial \psi_{\mathrm{m}}}{\partial R} \quad \text { at }(\rho, z) \neq(R, Z)
$$

The second of the key steps of Dyson' s technique is to invoke this identity to replace the combination of second derivatives by single first derivative. The importance of this step for promoting cancelation of terms cannot be overemphasized. Without the help from (13), a flood of terms become uncontrollable.

We introduce local cylindrical coordinates $(r, \theta)$ in the meridional plain by $(\hat{x}, \hat{z})=(r \cos \theta, r \sin \theta)$. The radius $r$ is the shortest distance from the given point $\boldsymbol{x}$ to the vortex loop. Integration of (12) is 
implemented, in terms of the first and the second complete elliptic integrals [5]. Use of the asymptotic formulas of the complete elliptic integrals for modulus close to unity leads us to the near field of $\psi_{\mathrm{m}}$, valid for $\sigma \ll r \ll R$, as

$$
\begin{aligned}
\psi_{\mathrm{m}}= & -\frac{R}{2 \pi}\left\{\log \left(\frac{8 R}{r}\right)-2+\frac{r}{2 R}\left[\log \left(\frac{8 R}{r}\right)-1\right] \cos \theta\right. \\
& \left.+\frac{r^{2}}{2^{4} R^{2}}\left(\left[2 \log \left(\frac{8 R}{r}\right)+1\right]-\left[\log \left(\frac{8 R}{r}\right)-2\right] \cos 2 \theta\right)+\cdots\right\},
\end{aligned}
$$

(see ref. [8]). The exponential decrease of coefficients, that is, in increase power of $2^{-1}$, makes the higher-order formula of translation speed applicable to fat cores.

We anticipate, for the vorticity $\zeta(x, z)=\zeta_{0}(r)+\zeta_{11}^{(1)} \cos \theta+\left(\zeta_{0}^{(2)}+\zeta_{21}^{(2)} \cos 2 \theta\right)+\cdots$, compatible with the Euler and the Navier-Stokes equations. For the coefficients $\zeta_{i j}^{(k)}$, being functions of $r, k$ designates the order of perturbation and $i$ labels the Fourier mode with $j=1$ and 2 corresponding to $\cos i \theta$ and $\sin i \theta$ respectively. With this form, we perform integration with respect to $\hat{x}^{\prime}$ and $\hat{z}^{\prime}$ in (11) and simplify the resulting expression with the help of (13). Substitution from (14) yields the asymptotic form of the Biot-Savart law, whose expression is, if we retain to first order in $\varepsilon=\sigma / R$ say, as

$\psi=-\frac{\Gamma R_{0}}{2 \pi}\left[\log \left(\frac{8 R_{0}}{r}\right)-2\right]+\left\{-\frac{\Gamma}{4 \pi}\left[\log \left(\frac{8 R_{0}}{r}\right)-1\right] r+\frac{d_{1}}{r}\right\} \cos \theta+\cdots$,

where $\Gamma=2 \pi \int_{0}^{\infty} r \zeta_{0} \mathrm{~d} r$, and the strength $d_{1}$ of the dipole is connected with $\zeta_{0}$ and $\zeta_{11}^{(1)}$.

The asymptotic form (15) serves as the inner limit of the outer solution and thus supplies the matching condition on the inner solution. Given $\zeta_{0}$, the profiles of $\zeta_{11}^{(1)}, \zeta_{0}^{(2)}$ and $\zeta_{21}^{(2)}$ should be determined by solving the Navier-Stokes or Euler equations in the inner region. When the vorticity is confined in the core, the expression (15) is validated to the edge of the core, and the translation speed is determined by imposing the condition that the boundary is coincident with a streamline. This was the approach taken by the successors of Kelvin [6-8]. To recover Kelvin's formula, representation (15), valid to $O(\varepsilon)$, is sufficient, but J. J. Thomson [6] overlooked the contribution from the local dipole field which includes $d_{1}$. This dipole field stems from an effective vortex pair generated by vortex-line stretching on the convex side and contraction on the concave side when a strait vortex tube is bent into a torus, which has ability to derive itself. For Kelvin's vortex ring, $d_{1}=3 \sigma^{2} \Gamma /\left(16 \pi R_{0}\right)$ and this is equivalent to the flow field around a cylinder of radius $\sigma$ moving in the $z$ direction with the speed $\Gamma /\left(4 \pi R_{0}\right) \times 3 / 4[12,13]$. This contribution repairs J. J. Thomson's results.

For a general distribution of vorticity, to carry out the inner expansion along with the extension of (15) is a rather cumbersome task. The treatment initiated by Helmholtz sidesteps the inner solution to a great extent, which is the topic of the following section. We note in passing that Dyson's technique has been extended to a helical vortex tube [38] and to a general three-dimensional vortex tube [39].

\section{Helmholtz-Lamb's method}

Helmholtz-Lamb's method is very efficient in that it allows us to reach the correction of $O\left(\varepsilon^{3}\right)$ to translation speed of a vortex ring without having to derive the $O\left(\varepsilon^{3}\right)$ velocity field. Rott-Cantwell [37] gave a lucid account of this method.

Under the boundary condition $|\boldsymbol{A}| \rightarrow 0$ as $|\boldsymbol{x}| \rightarrow \infty$, on the vector potential $\boldsymbol{A}$, the total kinetic energy $H$ of fluid filling an unbounded space, defined by $H=1 / 2 \int \boldsymbol{u}^{2} \mathrm{~d} V$, has a representation, for the axisymmetric flow, of

$H=\frac{1}{2} \iiint \boldsymbol{\omega} \cdot \boldsymbol{A} \mathrm{d} V=-\pi \iint \zeta \psi \mathrm{d} A\left(=-\pi \iint \zeta \psi \mathrm{d} \rho \mathrm{d} z\right)$.

The hydrodynamic impulse (6) is reduced to

$\boldsymbol{P}=P_{z} \boldsymbol{e}_{z} ; \quad P_{z}=\pi \iint \zeta \rho^{2} \mathrm{~d} A$. 
Remember that the impulse is a constant even in the presence of viscosity $[1,5,19]$.

Helmholtz [4] introduced the vorticity centroid

$Z=\iint \zeta \rho^{2} z \mathrm{~d} A / \iint \zeta \rho^{2} \mathrm{~d} A$,

and thought of its time derivative as the traveling speed of the vortex ring. By virtue of constancy of (17) and of the vorticity flux across a material surface whose local form is $\zeta \mathrm{d} A$ [25-27], the differentiation of (18) in time $t$ immediately yields the traveling speed $U=\mathrm{d} Z / \mathrm{d} t$ in the form:

$U=\iint\left(u_{z} \zeta \rho^{2}+2 u_{\rho} \zeta z \rho\right) \mathrm{d} A / \iint \zeta \rho^{2} \mathrm{~d} A$.

It was verified that the viscous diffusion of vorticity does not alter this form [16,37]. Two alternative representations of energy $H=\iiint \boldsymbol{\omega} \cdot \boldsymbol{A} \mathrm{d} V / 2=\iiint \boldsymbol{u} \cdot(\boldsymbol{x} \times \boldsymbol{\omega}) \mathrm{d} V$ reads, for the axisymmetric flow[4]

$-\frac{1}{2} \iint \psi \zeta \mathrm{d} A=\iint\left(u_{z} \rho^{2}-u_{\rho} z \rho\right) \zeta \mathrm{d} A$.

This is used to eliminate the integral $\int u_{z} \zeta \rho^{2} \mathrm{~d} A$ from (19), leaving Helmholtz-Lamb's identity

$U \iint \zeta \rho^{2} \mathrm{~d} A=-\frac{1}{2} \iint \psi \zeta \mathrm{d} A+3 \iint \rho z u_{\rho} \zeta \mathrm{d} A$.

It is noteworthy that the derivation does not depend much on the detail of the dynamics, and hence (21) is applicable to a wide class of solutions. Helmholtz-Lamb's identity (21) and Rott-Cantwell's identity (19) both require the knowledge of velocity field in the core or the inner solution. We recall the asymptotic solution of the Euler or the Navier-Stokes equations at large Reynolds numbers [12] in the following section and at small Reynolds numbers [34] in Sect. 6.

\section{High-Reynolds-number vortex ring}

The inner solution for steady motion of a vortex ring or quasi-steady motion, in the presence of viscosity, is found by solving the Euler or the Navier-Stokes equations, subject to the matching condition (15), in powers of the small parameter $\varepsilon$ [12]. This is then substituted into (21). In the sequel we give an outline of evaluating (21) to obtain the third-order correction to the translation speed. The detailed procedure of calculating integrals in (21) is presented in the forthcoming paper [40].

To work out the inner solution, we introduce the relative velocity $\tilde{\boldsymbol{u}}$ in the meridional plane by $\boldsymbol{u}=\tilde{\boldsymbol{u}}+(\dot{R}, \dot{Z})$. Here a dot stands for differentiation with respect to time. Let us nondimensionalize the inner variables. The radial coordinate is normalized by the core radius $\varepsilon R_{0}(=\sigma)$ and the local velocity $(u, v)$, relative to the moving frame, by the maximum velocity $\Gamma /\left(\varepsilon R_{0}\right)$. In view of $(2)$, the normalization parameter for the ring speed $(\dot{R}(t), \dot{Z}(t))$, the slow dynamics, should be $\Gamma / R_{0}$. The suitable dimensionless inner variables are thus defined as

$r^{*}=r / \varepsilon R_{0}, \quad t^{*}=t / \frac{R_{0}}{\Gamma}, \quad \psi^{*}=\frac{\psi}{\Gamma R_{0}}, \quad \zeta^{*}=\zeta / \frac{\Gamma}{R_{0}^{2} \varepsilon^{2}}, \quad \tilde{\boldsymbol{u}}^{*}=\tilde{\boldsymbol{u}} / \frac{\Gamma}{R_{0} \varepsilon}, \quad\left(\dot{R}^{*}, \dot{Z}^{*}\right)=(\dot{R}, \dot{Z}) / \frac{\Gamma}{R_{0}}$

The difference in normalization between the last two of (22) should be kept in mind. Correspondingly to (22), the kinetic energy (16) and the hydrodynamic impulse (17) are normalized as $H^{*}=$ $H / \Gamma^{2} R_{0}, P_{z}^{*}=P_{z} / \Gamma R_{0}^{2}$. Hereinafter we drop the superscript ${ }^{*}$ for dimensionless variables. Dimensionless form of the radial position $R$ of the core center is $R=1+\varepsilon^{2} R^{(2)}+O\left(\varepsilon^{3}\right)$. We can maintain the first term to be unity by adjusting disposable parameters, bearing with the origin of coordinates, in the first-order field [12]. The second-order correction $\varepsilon^{2} R^{(2)}$ is tied with the viscous expansion.

A glance at the Euler or the Navier-Stokes equations shows that the dependence, on $\theta$, of the solution in a power series in $\varepsilon$ is

$$
\begin{aligned}
\psi & =\psi^{(0)}(r)+\varepsilon \psi_{11}^{(1)}(r) \cos \theta+\varepsilon^{2}\left[\psi_{0}^{(2)}(r)+\psi_{21}^{(2)}(r) \cos 2 \theta\right]+O\left(\varepsilon^{3}\right), \\
\zeta & =\zeta^{(0)}(r)+\varepsilon \zeta_{11}^{(1)}(r) \cos \theta+\varepsilon^{2}\left[\zeta_{0}^{(2)}(r)+\zeta_{21}^{(2)}(r) \cos 2 \theta\right]+O\left(\varepsilon^{3}\right) .
\end{aligned}
$$


Upon substitution from $(23)$ and $(24)$, we obtain a representation, to $O\left(\varepsilon^{2}\right)$ in dimensionless form, $H=H^{(0)}+\varepsilon^{2} H^{(2)}$ and $P_{z}=P^{(0)}+\varepsilon^{2} P^{(2)}$ of the kinetic energy and the hydrodynamic impulse, as

$H^{(0)}=-2 \pi^{2} \int_{0}^{\infty} r \zeta^{(0)} \psi^{(0)} \mathrm{d} r, \quad H^{(2)}=-2 \pi^{2} \int_{0}^{\infty} r\left(\frac{1}{2} \zeta_{11}^{(1)} \psi_{11}^{(1)}+\zeta^{(0)} \psi_{0}^{(2)}+\zeta_{0}^{(2)} \psi^{(0)}\right) \mathrm{d} r$,

$P^{(0)}=\pi, \quad P^{(2)}=\pi\left(2 R^{(2)}-4 \pi d^{(1)}\right)$,

where $d^{(1)}=d_{1} /\left(\Gamma \sigma^{2}\right)$ is the dimensionless strength of dipole.

Evaluation of (25) and (26) is relatively easy as these does not include the quadrupole field $\psi_{21}^{(2)}$ and $\zeta_{21}^{(2)}$. Given $\zeta^{(0)}$ to $O\left(\varepsilon^{0}\right)$, the azimuthal velocity to $O\left(\varepsilon^{0}\right)$ satisfies $v^{(0)}=-\partial \psi^{(0)} / \partial r$, and the Stokes streamfunction complying with $(15)$ is, to $O\left(\varepsilon^{0}\right)$,

$\psi^{(0)}=-\int_{0}^{r} v^{(0)}\left(r^{\prime}\right) \mathrm{d} r^{\prime}+\lim _{r \rightarrow \infty}\left\{\int_{0}^{r} v^{(0)}\left(r^{\prime}\right) \mathrm{d} r^{\prime}-\frac{1}{2 \pi}\left[\log \left(\frac{8}{\varepsilon r}\right)-2\right]\right\}$.

Without viscosity, the vorticity profile $\zeta^{(0)}$ may be taken to be arbitrary, but viscosity plays the role of selecting its functional form [15]. It is expedient to handle the streamfunction $\tilde{\psi}$ for the flow relative to the coordinates moving with the same speed $\dot{Z}$ as the vortex ring along the $z$-direction, namely, $\psi=-\dot{Z} \rho^{2} / 2+\tilde{\psi}$. The first-order solution comprises a dipole field. Denoting the dipole coefficient of the streamfunction for the flow, relative to the moving frame, to be $\tilde{\psi}_{11}^{(1)}=\psi_{11}^{(1)}+r \dot{Z}^{(0)}$, the coefficient function $\tilde{\psi}_{11}^{(1)}$ is given by

$\tilde{\psi}_{11}^{(1)}=-v^{(0)}\left\{\frac{r^{2}}{2}+\int_{0}^{r} \frac{\mathrm{d} r^{\prime}}{r^{\prime}\left[v^{(0)}\left(r^{\prime}\right)\right]^{2}} \int_{0}^{r^{\prime}} r^{\prime \prime}\left[v^{(0)}\left(r^{\prime \prime}\right)\right]^{2} \mathrm{~d} r^{\prime \prime}\right\}+c_{11}^{(1)} v^{(0)}$

where $c_{11}^{(1)}$ is a disposable parameter tied with choice of the origin $r=0$ of the local coordinates. The vorticity is found from $\zeta_{11}^{(1)}=a \tilde{\psi}_{11}^{(1)}+r \zeta^{(0)}$ with $a(r, t)=-1 / v^{(0)}\left(\partial \zeta^{(0)} / \partial r\right)$. The Fourier coefficient $\tilde{\psi}_{0}^{(2)}(r)$ of the monopole component of $O\left(\varepsilon^{2}\right)$, relative to the moving coordinate frame, defined by $\tilde{\psi}_{0}^{(2)}=\psi_{0}^{(2)}+\dot{Z}^{(0)} r^{2} / 4$ is written in terms of $v^{(0)}, \tilde{\psi}_{11}^{(1)}$ and $\zeta_{0}^{(2)}$. The $O\left(\varepsilon^{2}\right)$ monopole component $\zeta_{0}^{(2)}$ of vorticity obeys a heat-conduction equation with source terms [12].

A steady inviscid vortex ring or a quasi-steady viscous vortex corresponds to a state of the maximum energy and this critical states favors core shape with back-to-fore symmetry [26,41]. This symmetry, $\zeta(\rho,-\hat{z})=-\zeta(\rho, \hat{z})$ and $u_{\rho}(\rho,-\hat{z})=-u_{\rho}(\rho, \hat{z})$, simplifies the last integral in $(21)$ to

$J=\iint \rho z u_{\rho} \zeta \mathrm{d} A=\int_{0}^{2 \pi} \int_{0}^{\infty} r \sin \theta\left(\sin \theta \frac{\partial \tilde{\psi}}{\partial r}+\frac{\cos \theta}{r} \frac{\partial \tilde{\psi}}{\partial \theta}\right) \zeta r \mathrm{~d} r \mathrm{~d} \theta$.

Substituting from $(23)$ and $(24),(29)$ becomes $J=J^{(0)}+\varepsilon^{2} J^{(2)}$, to $O\left(\varepsilon^{2}\right)$, where

$J^{(0)}=-\pi \int_{0}^{\infty} r^{2} v^{(0)} \zeta^{(0)} \mathrm{d} r=\frac{1}{8 \pi}$
$J^{(2)}=-\pi \int_{0}^{\infty} r^{2}\left[v^{(0)}\left(\zeta_{0}^{(2)}-\frac{1}{2} \zeta_{21}^{(2)}\right)+\frac{1}{4}\left(\frac{\tilde{\psi}_{11}^{(1)}}{r}-\frac{\partial \tilde{\psi}_{11}^{(1)}}{\partial r}\right) \zeta_{11}^{(1)}+\left(\frac{\tilde{\psi}_{21}^{(2)}}{r}+\frac{1}{2} \frac{\partial \tilde{\psi}_{21}^{(2)}}{\partial r}-\frac{\partial \tilde{\psi}_{0}^{(2)}}{\partial r}\right) \zeta^{(0)}\right] \mathrm{d} r$.

The leading-order term $H^{(0)}$ of energy is evaluated with ease, by introducing (27) into (25), which is expressed, in dimensional variables, as

$H_{0} / \Gamma^{2}=\frac{1}{2} R_{0}\left\{\log \left(\frac{8 R_{0}}{\sigma}\right)+A-2\right\}$,

where $H_{0}=\Gamma^{2} R_{0} H^{(0)}$ and $A$ is defined by (3). This expression, along with $P^{(0)}$ and $J^{(0)}$, gives rise to Fraenkel-Saffman's formula (3), via (21). The third-order correction $U_{2}$ to the translation speed of the vortex ring requires evaluation of $H^{(2)}$ and $J^{(2)}$. Evaluation of $(31)$ is rather involved as it includes $\zeta_{21}^{(2)}$ and $\tilde{\psi}_{21}^{(2)}$, the quadrupole field of $O\left(\varepsilon^{2}\right)$. But (31) is somehow simplified by use of equation 


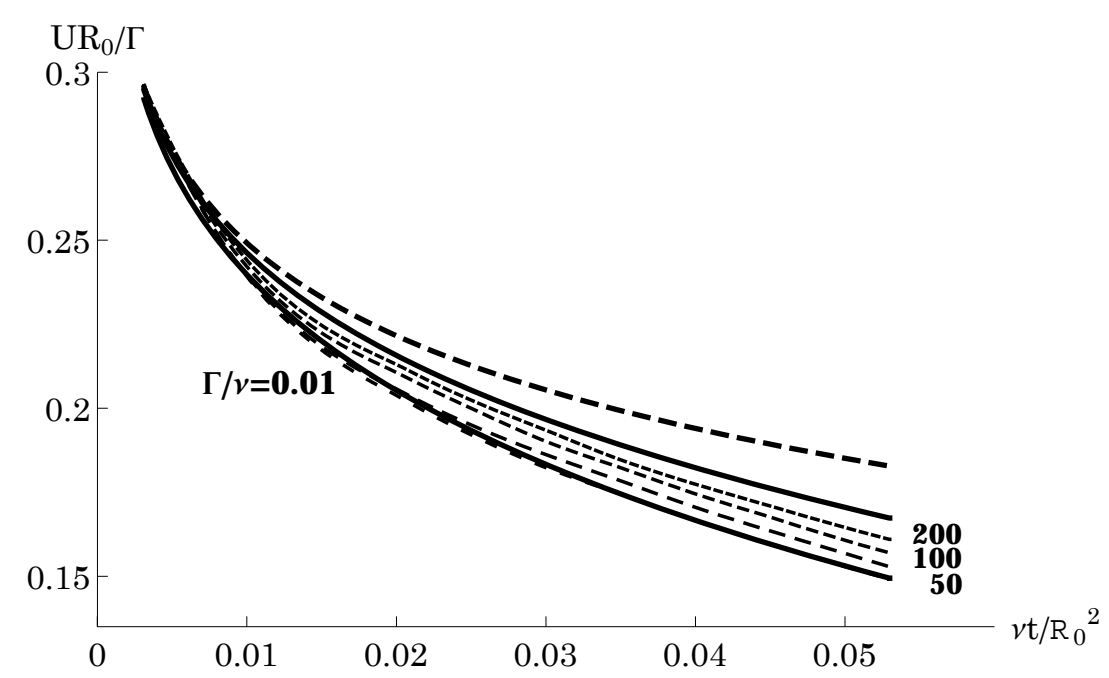

Fig. 2 Variation of speed of a viscous vortex ring with time. The upper and lower solid lines are the highand low-Reynolds number asymptotics (36) and (44), respectively, while the thick dashed line is the Saffman's formula (5). The dashed lines are the values read off from the graph of numerical simulations [42].

governing $\tilde{\psi}_{21}^{(2)}$ and the relation between $\tilde{\psi}_{21}^{(2)}$ and $\zeta_{21}^{(2)}$. For an inviscid vortex ring in steady motion, $R_{2}=R_{0} \varepsilon^{2} R^{(2)} \equiv 0$ without loss of generality, and, after some manipulations, we arrive at

$U_{2}=\frac{1}{R_{0}^{3}}\left\{\frac{d_{1}}{2}\left[\log \left(\frac{8 R_{0}}{\sigma}\right)-2\right]-\pi \Gamma B+\frac{\pi}{2 \Gamma} \int_{0}^{\infty} r^{4} \zeta_{0} v_{0} \mathrm{~d} r\right\}$

where $v_{0}=\Gamma v^{(0)} / \sigma$ and $\zeta_{0}=\Gamma \zeta^{(0)} / \sigma^{2}$ are dimensional variables, and

$B=\lim _{r \rightarrow \infty}\left\{\frac{1}{\Gamma^{2}} \int_{0}^{r} r^{\prime} v_{0} \tilde{\psi}_{11}^{(1)} \mathrm{d} r^{\prime}+\frac{r^{2}}{16 \pi^{2}}\left[\log \left(\frac{r}{\sigma}\right)+A\right]+\frac{d_{1}}{2 \pi \Gamma} \log \left(\frac{r}{\sigma}\right)\right\}$.

This is an extension, to $O\left(\varepsilon^{3}\right)$, of Fraenkel-Saffman's formula (3). The same formula was reached by way of the variational principle [14] as will be touched upon in the following section.

Even if viscosity is switched on, the higher-order asymptotics $U_{2}$ is not invalidated at a large Reynolds number. Taking, as the initial condition, a circular line vortex of radius $R_{0}$,

$\zeta(\rho, z, 0)=\Gamma \delta\left(\rho-R_{0}\right) \delta(z-Z) \quad$ at $t=0$

the leading-order vorticity $\zeta_{0}$ is given by $(4)[16,15]$, and the inhomogeneous heat-conduction equation governing $\zeta_{0}^{(2)}$ becomes tractable, with an introduction of similarity variables. The parameters $c_{11}^{(1)}$ in (28) and $R_{2}$, both being functions of $t$, play a common role of specifying the radial position of the ring at $O\left(\varepsilon^{2}\right)$ relative to $R_{0}$. This redundancy is removed, for instance, by taking $c_{11}^{(1)} \equiv 0$. Thus we are led to an extension of Saffman's formula (5) in the form

$U \approx \frac{\Gamma}{4 \pi R_{0}}\left\{\log \left(\frac{4 R_{0}}{\sqrt{\nu t}}\right)-0.55796576-3.6715912 \frac{\nu t}{R_{0}^{2}}\right\}$.

Fig. 2 displays the comparison of the asymptotic formula (36) with a direct numerical simulation of the axisymmetric Navier-Stokes equations [42]. The normalized speed $U R_{0} / \Gamma$ of the ring is drawn as a function of normalized time $\nu t / R_{0}^{2}$ for its small values. The upper thick solid line is our formula (36), and the thick broken line is the first-order truncation (5). The dashed lines are the results of the numerical simulations, attached with the circulation Reynolds number $\Gamma / \nu$, ranging from 0.01 to 200 . Augmented only with a single correction term, (33) appears to furnish a close upper bound on the translation speed. Notably, the large-Reynolds-number asymptotic formula (36) compares fairly well with the numerical result of even moderate and small Reynolds numbers. 
Constancy of the hydrodynamic impulse (17), regardless of the presence of viscosity, provides us with a short-cut to reach the radial motion of the ring; the third-order motion $R^{(2)}$ is gained solely from the first-order velocity field [12]. For the initial $\delta$-function core (35), the peak-vorticity circle of radius $R_{p}(t)$ and the vorticity centroid $R_{c}$ in the radial direction expands, respectively, as [12]

$R_{p} \approx R_{0}+4.5902739 \frac{\nu t}{R_{0}}, \quad R_{c}=\frac{\pi}{P_{z}} \iint \rho^{3} \zeta r \mathrm{~d} r \mathrm{~d} \theta \approx R_{0}+\frac{3 \nu t}{R_{0}}$.

The third-order formulas (36) and (37), intended for $\sqrt{\nu t} \ll R_{0}$, has a wider applicability than envisaged, but ceases to be valid when the core is so swollen as to touch itself and cancellation of vorticity takes place on the symmetry axis $(\rho=0)$. Saffman [16] made a judicious treatment of simplifying the Navier-Stokes equations for estimation of the traveling speed of a vortex ring valid after $\nu t \approx R_{0}^{2}$, and obtained, with use of some constant $k$ and $k^{\prime}$.

$R^{2}=R_{0}^{2}+k^{\prime} \nu t, \quad U=\frac{P_{z}}{k}\left(R_{0}^{2}+k^{\prime} \nu t\right)^{-3 / 2}$.

This tends, at $\nu t \gg R_{0}^{2}$, to Rott-Cantwell's decaying law (8). Saffman's matured-stage formula (38) exhibits a good fit to an experimental measurement of using the DPIV [43]. The measurements of location of peak vorticity tells $k^{\prime}=7.8$. If the small-time asymptotics (37) is translated into (38), $k^{\prime}=9.1805478$. The agreement is acceptable.

Although Helmholtz-Lamb's method (21) saves the labor, the integral $J$ in (21), with including $\zeta_{21}^{(2)}$ and $\zeta_{21}^{(2)}$, stands as an obstacle. The variational principle (7), which comprises only the total energy $H$ and the impulse $P_{z}$, dispenses with $\zeta_{21}^{(2)}$ and $\zeta_{21}^{(2)}$. A further simplification is achieved by relying on the variational principle with kinematic constraints.

\section{Kelvin-Benjamin's variational principle}

It is well known that a stationary configuration of vorticity, embedded in an inviscid incompressible fluid, is realizable as an extremal of energy on an iso-vortical sheet [24,25]. An iso-vortical sheet comprises volume-preserving diffeomorphisms, or smooth maps of fluid particles, with vorticity frozen into the fluid. Put it in another way, the critical state is sought among the class of $\boldsymbol{\omega}$ that is reached by rearrangement of the initial distribution. Extending this conditional variational principle to a moving state, Benjamin [26] stated that an axisymmetric vortex ring moving steadily in an inviscid incompressible fluid is realizable as the maximum state of the kinetic energy $H$ on an iso-vortical sheet, subject to the constraint of constant hydrodynamic impulse (6). An upper bound of the kinetic energy, supplied by a topological invariant $[27,28]$, guarantees the existence of the maximum state. When translated into three dimensions, Kelvin-Benjamin's principle takes the form of (7) with constant vector $\boldsymbol{U}$ playing the role of the Lagrangian multipliers [41]. The restriction of axisymmetry can be lifted and (7) is extended to a stationary vorticity distribution in a steadily moving frame [14].

An iso-vortical sheet is of infinite dimension. A family of solutions of the Euler equations includes a few parameters. By imposing certain relations among these parameters, we can maintain the solutions on a single iso-vortical sheet, and the restricted family of the solutions constitutes a finite dimensional set on the sheet. Thus the traveling speed of a vortex ring may be calculable through (7). Dyson's vortex ring (2) was dealt with in this framework [22]. The same is true of Saffman's formula (5) [14], though excluded from the list of [18].

We pose, as a natural profile of local velocity field featuring a vortex ring,

$v_{0}(r)=-\frac{\Gamma}{2 \pi r} f\left(\frac{r}{\sigma}\right), \quad \zeta_{0}=\frac{\Gamma}{2 \pi r} \frac{\mathrm{d}}{\mathrm{d} r} f\left(\frac{r}{\sigma}\right) ; \quad f(\xi)=O\left(\xi^{2}\right)$ as $\xi \rightarrow 0, \quad f(\xi) \rightarrow 1$ as $\xi \rightarrow \infty$.

where $f$ is an arbitrary function, though subjected to the above boundary conditions. The parameter $\sigma$ introduces the scale for the core thickness. Suppose that the fluid particles occupying a toroidal region of radius $r$ around the center circle of radius $R$ is mapped to another toroidal region of radius $\hat{r}$ around the center circle of radius $\hat{R}$. To maintain these flow field on an iso-vortical sheet, it is necessary for the local circulation along any material loop to remain unchanged $[25,27,28]$. Preservation of material volume enforces $2 \pi^{2} r^{2} R=2 \pi^{2} \hat{r}^{2} \hat{R}, 2 \pi^{2} \sigma^{2} R=2 \pi^{2} \hat{\sigma}^{2} \hat{R}$, from which follows $r / \sigma=\hat{r} / \hat{\sigma}$. Consequently, the local circulation around the circle of radius $r, \Gamma(r)=2 \pi \int_{0}^{r} \zeta_{0}\left(r^{\prime}\right) r^{\prime} \mathrm{d} r^{\prime}=\Gamma f(r / \sigma)$, is made invariant: 
$\Gamma(r)=\Gamma(\hat{r})$. Under an infinitesimal perturbation of $R \rightarrow \hat{R}=R+\delta R, \sigma \rightarrow \hat{\sigma}=\sigma+\delta \sigma$, with $R=R_{0}+R_{2}$, (5) demands that, at each order, $\sigma^{2} R_{0}=$ const. and $\sigma^{2} R_{2}=$ const., and therefore that $2 \delta \sigma / \sigma=-\delta R_{0} / R_{0}=-\delta R_{2} / R_{2}$. We can show that, under this perturbation, $\hat{A}=A+O\left((\delta R)^{2}\right)$. In view of these constraints, the variation of (32) with respect to an iso-vortical perturbation becomes

$\delta H_{0}=\frac{\Gamma^{2}}{2}\left[\log \left(\frac{8 R_{0}}{\sigma}\right)+A-\frac{1}{2}\right] \delta R_{0}$.

The variation of the leading term of impulse $P_{0}=\Gamma \pi R_{0}^{2}$ is $\delta P_{0}=2 \pi \Gamma R_{0} \delta R_{0}$, and application of (7) restores Fraenkel-Saffman's formula (3).

This principle is extensible to higher orders, whereby the $O\left(\varepsilon^{3}\right)$ corrections (33) and (36) are produced [14]. Mohseni [44] devised an efficient algorithm of combining (7) with the slug model to estimate the translation velocity of fat vortex rings.

\section{Low-Reynolds-number vortex ring}

Saffman's second law (38) well describes the timewise variation of traveling speed after the matured stage $\left(\sqrt{\nu t} \geq R_{0}\right)$, by an adjustment of the disposable parameters $k$ and $k^{\prime}$. For $\sqrt{\nu t} \gg R_{0}$, (38) approaches Rott-Cantwell's decaying law (8) for which the velocity field is given by Phillips' spherical dipole [45], an exact solution of the Stokes equations. Given an initial delta function core (35), the early-time behavior (5) of the translation speed is common to $O(\varepsilon)$, independently of the Reynolds number $\Gamma / \nu$. At low Reynolds numbers, there is a solution that is valid over the whole time range $(t \geq 0)$, illustrating how the early time behavior (5) of a thin core crosses over to (8) [33,34].

We suppose that the vorticity is governed by the Stokes equations. Their solution, subject to the initial condition $\zeta_{0}(z, \rho, 0)=\Gamma_{0} \delta(z) \delta\left(\rho-R_{0}\right)$, with $\Gamma_{0}$ being a constant, is

$$
\zeta=\frac{\Gamma_{0} R_{0}}{4 \sqrt{\pi}(\nu t)^{3 / 2}} \exp \left(-\frac{z^{2}+\rho^{2}+R_{0}^{2}}{4 \nu t}\right) I_{1}\left(\frac{R_{0} \rho}{2 \nu t}\right)
$$

where $I_{1}$ is the first-kind modified Bessel function of order unity. This expression was first found mathematically as a similarity solution [46] and was then given an interpretation in the context of evolution of a viscous vortex ring $[33,34]$. The total vorticity in the half meridional plane $(\rho \geq 0)$ decreases as $\Gamma=\Gamma_{0}\left[1-\exp \left(-R_{0}^{2} / 4 \nu t\right)\right]$, and the hydrodynamic impulse is $P_{z}=\pi R_{0}^{2} \Gamma_{0}$. The corresponding Stokes streamfunction is

$\Psi=-\frac{\Gamma_{0} R_{0} \rho}{4} \int_{0}^{\infty}\left[e^{p z} \operatorname{erfc}\left(\frac{2 p \nu t+z}{2 \sqrt{\nu t}}\right)+e^{-p z} \operatorname{erfc}\left(\frac{2 p \nu t-z}{2 \sqrt{\nu t}}\right)\right] J_{1}\left(p R_{0}\right) J_{1}(p \rho) \mathrm{d} p$.

The behavior of (41) and (42) at large times $\left(\sqrt{\nu t} \gg R_{0}\right)$ coincides with that of Phillips' solution. Upon substitution from (41) and (42), (21) gives rise to the desired formula for the translation velocity:

$$
\begin{aligned}
U= & \frac{\Gamma_{0} R_{0}^{2}}{96 \sqrt{2 \pi}(\nu t)^{3 / 2}}\left\{{ }_{2} F_{2}\left(\frac{3}{2}, \frac{3}{2} ; \frac{5}{2}, 3 ;-\frac{R_{0}^{2}}{2 \nu t}\right)-\frac{36}{5}{ }_{2} F_{2}\left(\frac{3}{2}, \frac{5}{2} ; 2, \frac{7}{2} ;-\frac{R_{0}^{2}}{2 \nu t}\right)\right. \\
& \left.+\frac{72 \nu t}{R_{0}^{2}} \exp \left(-\frac{R_{0}^{2}}{4 \nu t}\right) I_{1}\left(\frac{R_{0}^{2}}{4 \nu t}\right)\right\},
\end{aligned}
$$

where ${ }_{2} F_{2}$ is the generalized hypergeometric function [33]. One of advantages of this representation is to use the expansion of ${ }_{2} F_{2}$ at small arguments and to exploit the analytic continuation to derive asymptotic expansions at large arguments. Using the asymptotic form of ${ }_{2} F_{2}$ for negative large and small values of the argument, we can deduce early- and long-time behavior of (43) as follows [34].

$$
\begin{aligned}
U & \approx \frac{\Gamma_{0}}{4 \pi R_{0}}\left\{\log \left(\frac{4 R_{0}}{\sqrt{\nu t}}\right)-0.55796576-4.5\left[\log \left(\frac{4 R_{0}}{\sqrt{\nu t}}\right)-1.0579658\right] \frac{\nu t}{R_{0}^{2}}\right\} \quad \text { for } \sqrt{\nu t} \ll R_{0}, \\
& =\frac{7 P_{z}}{240 \sqrt{2}(\pi \nu t)^{3 / 2}}\left\{1-\frac{33}{196} \frac{R_{0}^{2}}{\nu t}+\frac{125}{6272}\left(\frac{R_{0}^{2}}{\nu t}\right)^{2}+O\left(\left(\frac{R_{0}^{2}}{\nu t}\right)^{3}\right)\right\} \quad \text { for } \sqrt{\nu t} \gg R_{0} .
\end{aligned}
$$


The translation speed (43) based on the Stokesian dynamics of vorticity poses the strict lower bound on $U$. Figure 2 confirms this at small values of $t$. Fair agreement is observed between (44) and the numerical result of the Reynolds number $\Gamma / \nu=0.01$, the lowermost dashed line. Notice that the traveling speed is not very sensitive to $\Gamma / \nu$.

The large-time behavior (45) gives corrections to Rott-Cantwell's formula (8). Comparison of the first two terms of (45) with those of Saffman's second formula (38) as expanded in $R_{0}^{2} / \nu t$ yields $k=1320 \sqrt{11} \pi^{3 / 2} / 2401 \approx 10.15, k^{\prime}=98 / 11 \approx 8.909$. With this choice, $(38)$ furnishes an excellent interpolation formula between (44) and (45), and exhibits a fairly good approximation to (43) even at small values of $\nu t / R_{0}^{2}$.

Another advantage of the representation (43) is that we can calculate, in a tidy form, the distance $s(t)=\int_{0}^{t} U(\tau) \mathrm{d} \tau$ traversed over time $t$ by the viscous vortex ring [34]. This expression gives a partial answer to the question of the inverse problem raised in Sect. 1. When does a vortex ring arrive at a specified point? The traveling speed slows down with $t$, and ultimately decreases to zero in proportion to $t^{-3 / 2}$ as is seen from (8). This implies that the vortex ring cannot be freely sent to remote regions. Taking the limit $t \rightarrow \infty$ of $s(t)$ shows that the distance $s_{\max }$ of the furthermost reach is

$s_{\max }=\frac{5 \Gamma_{0} R_{0}}{24 \pi \nu}=\frac{5 P_{z}}{24 \pi^{2} R_{0} \nu} \approx 0.066314560 \frac{\Gamma_{0} R_{0}}{\nu}$.

The maximum reach $s_{\max }$ extends in inversely proportion to $\nu$. Given the impulse $P_{z}, s_{\max }$ is larger as the initial ring radius $R_{0}$ is smaller.

The vortex bubble is a region encircling the vortex core bounded by a surface of zero streamfunction of the flow relative to the ring motion. For an inviscid vortex ring, the volume of the vortex bubble is a function of the Stokes streamfunction for the relative flow and is called the vortex-ring signature $[27,47]$. The vortex-ring signature of our low-Reynolds-number solution, bears, with an appropriate normalization, some resemblance with that of the direct numerical simulation of the axisymmetric Navier-Stokes equations [32]. Recently, a family of similarity solutions is found [48], that includes (41) and (42) as an extreme and may describe a turbulent vortex ring in the other extreme.

\section{Summary}

Vortex rings stimulated development of mathematical machinery. This article has described a partial history of this development as exemplified by Helmholtz-Lamb identity for the movement of the vorticity centroid, Dyson's technique for asymptotic expansions of the Biot-Savart law, and Kelvin's variational principle as augmented with the effect of motion of a vortical region by Benjamin. Helmholtz's seminal paper [4] illuminated the preservation of topology of the vorticity field. This topological idea has been rediscovered in various guises over and over again and has played a vital role in developing theories of vortex dynamics, including vortex-ring motion.

Mathematical labor to reach the same formula for the speed of a vortex ring dramatically decreases in order of the method of matched asymptotic expansions [12], Helmholtz-Lamb's method and the variational principle. By appealing to these efficient methods, we have succeeded in achieving higher-order extension of Fraenkel-Saffman's and Saffman's formulas, which are applicable to fat cores. Hopefully these methods carry over to helical vortex tubes with allowance made for torsion and the rotation of the system ( $c f$. [38]). For low-Reynolds-number motion, exploiting formulas associated with the generalized hypergeometric functions is advantageous to extract rich information.

Acknowledgements This article is based on collaboration with H. K. Moffatt and F. Kaplanski. I owe much to them. This work was partially supported by a Grant-in-Aid for Scientific Research from the JSPS (Grant No. 19540406).

\section{References}

1. Batchelor, G. K.: An Introduction to Fluid Dynamics. Cambridge University Press (1967)

2. Okabe, J.: Essay on the occasion of retirement. [in Japanese]. Research Institute of Applied Mechanics, Kyushu University (1984)

3. Kosugi, T., Takamori, F. Hashiguchi, Takeda, T.: Design and consideration of air cannons for tactile display. In papers of the 13th Annual Meeting of the Japan Society of Virtual Reality [in Japanese], 401-404 (2008) 
4. Helmholtz, H. von.: Über integrale der hydrodynamischen gleichungen welche den wirbelbewegungen entsprechen. Crelles, J. 55, 25 (1858). English translation by Tait, P. G.: On integrals of the hydrodynamical equations which express vortex-motion. Phil. Mag. (4) 33, 485-512 (1867)

5. Lamb, H.: Hydrodynamics, Chap. 7. Cambridge University Press (1932)

6. Thomson, J. J.: A Treatise on the Motion of Vortex Rings. Mcmillan, London (1883)

7. Hicks, W. M.: Researches on the theory of vortex rings - part II. Phil. Trans. R. Soc. Lond. A 176, 725-780 (1885)

8. Dyson, F. W.: The potential of an anchor ring. - part II. Phil. Trans. R. Soc. Lond. A 184, 1041-1106 (1893)

9. Fraenkel, L. E.: On steady vortex rings of small cross-section in an ideal fluid. Proc. R. Soc. Lond. A 316, 29-62 (1970)

10. Fraenkel, L. E.: Examples of steady vortex rings of small cross-section in an ideal fluid. J. Fluid Mech. 51, 119-135 (1972)

11. Norbury, J.: A family of steady vortex rings. J. Fluid Mech. 57, 417-431 (1973)

12. Fukumoto, Y., Moffatt, H. K.: Motion and expansion of a viscous vortex ring. Part 1. A higher-order asymptotic formula for the velocity. J. Fluid Mech. 417, 1-45 (2000)

13. Fukumoto, Y.: Higher-order asymptotic theory for the velocity field induced by an inviscid vortex ring. Fluid Dyn. Res. 30, 65-92 (2002)

14. Fukumoto, Y., Moffatt, H. K.: Kinematic variational principle for motion of vortex rings. Physica D 237, 2210-2217 (2008)

15. Tung, C., Ting, L.: Motion and decay of a vortex ring. Phys. Fluids 10, 901-910 (1967)

16. Saffman, P. G.: The velocity of viscous vortex rings. Stud. Appl. Math. 49, 371-380 (1970)

17. Callegari, A. J., Ting, L.: Motion of a curved vortex filament with decaying vortical core and axial velocity. SIAM J. Appl. Maths 35, 148-175 (1978)

18. Donnelly, R. J.: Quantized Vortices in Helium II. Chap. 1. Cambridge University Press (1991)

19. Saffman, P. G.: Vortex Dynamics. Cambridge University Press (1992)

20. Shariff, K., Leonard, A.: Vortex rings. Annu. Rev. Fluid Mech. 24, 235-279 (1992)

21. Lim, T., Nickels, T.: Vortex rings. In: Green, S. I. (ed.) Fluid Vortices Kluwer, Dordrecht (1995)

22. Roberts, P. H., Donnelly, R. J.: Dynamics of vortex rings. Phys. Lett. A 31, 137-138 (1970)

23. Roberts, P. H.: A Hamiltonian theory for weakly interacting vortices. Mathematika 19, 169-179 (1972)

24. Kelvin, Lord: On the stability of steady and of periodic fluid motion. Phil. Mag. 23, 459-464 (1878)

25. Arnol'd, V. I.: Sur la géométrie différentielle des groupes de Lie de dimension infinie et ses applications á l'hydrodynamique des fluids parfaits. Ann. Inst. Fourier Grenoble 16, 319-361 (1966)

26. Benjamin, T. B.: The alliance of practical and analytical insights into the nonlinear problems of fluid Mechanics. Lecture Notes in Math. No. 503, 8-29, Springer-Verlag (1976)

27. Moffatt, H. K.: Structure and stability of solutions of the Euler equations: a lagrangian approach. Phil. Trans. R. Soc. Lond. A 333, 321-342 (1990)

28. Fukumoto, Y.: A unified view of topological invariants of fluid flows. Topologica 1, 003 (2008)

29. Jones, C. A., Roberts, P. H.: Motions in a Bose condensate: IV. Axisymmetric solitary waves. J. Phys. A: Math. Gen. 15, 2599-2619 (1982)

30. Maxworthy, T.: The structure and stability of vortex rings. J. Fluid Mech. 51, 15-32 (1972)

31. Dabiri, J., Gharib, M.: Fluid entrainment by isolated vortex rings. J. Fluid Mech. 511, 311-331 (2004)

32. Danaila, I., Hélie, J.: Numerical simulation of the postformation evolution of a laminar vortex rings. Phys. Fluids 20, 073602 (2008)

33. Kaplanski, F., Rudi, U.: A model for the formation of 'optimal' vortex rings taking into account viscosity. Phys. Fluids 17, 087101 (2005)

34. Fukumoto, Y., Kaplanski, F.: Global time evolution of an axisymmetric vortex ring at low Reynolds numbers. Phys. Fluids 20, 053103 (2008)

35. Kambe, T., Oshima, Y.: Generation and decay of viscous vortex rings. J. Phys. Soc. Japan 38, 271-280 (1975).

36. Rott, N., Cantwell, B.: The decays of a viscous vortex pair Phys. Fluids 31, 3213-3224 (1988)

37. Rott, N., Cantwell, B.: Vortex drift. I: Dynamic interpretation Phys. Fluids A 5, 1443-1450 (1993)

38. Fukumoto, Y., Okulov, V. L.: The velocity field induced by a helical vortex tube. Phys. Fluids 17, 107101 (2005)

39. Fukumoto, Y.: Three-dimensional motion of a vortex filament and its relation to the localized induction hierarchy. Euro. Phys. J. B 29, 167-171 (2002)

40. Fukumoto, Y., Moffatt, H. K.: in preparation (2009)

41. Burton, G. R.: Vortex-rings of prescribed impulse. Math. Proc. Camb. Phil. Soc. 134, 515-528 (2003)

42. Stanaway, S. K., Cantwell, B. J., Spalart, P. R.: A numerical study of viscous vortex rings using a spectral method. NASA Technical Memorandum 101041 (1988)

43. Weigand, A., Gharib, M.: On the evolution of laminar vortex rings. Exps. Fluids 22, 447-457 (1997)

44. Mohseni, K.: A formulation for calculating the translational velocity of a vortex rings or pair. Bioinsp. Biomim. 1, S57-S64 (2006)

45. Phillips, O. M.: The final period of decay of non-homogeneous turbulence. Proc. Camb. Phil. Soc. 252, Pt. 1, 135-151 (1956).

46. Kaltaev, A.: Investigation of dynamic characteristics of motion of a vortex ring of viscous fluid. [In Russian] In Continuum Dynamics. Kazah State University, Alma-Ata, 63 (1982)

47. Moffatt, H. K.: Generalised vortex rings with and without swirl. Fluid Dyn. Res. 3, 22-30 (1988)

48. Kaplanski, F., Sazhin, S. S., Fukumoto, Y., Begg, S., Heikal, M.: A generalised vortex ring model. J. Fluid Mech. in press (2009) 


\section{List of MI Preprint Series, Kyushu University}

The Grobal COE Program

Math-for-Industry Education \& Research Hub

MI

MI2008-1 Takahiro ITO, Shuichi INOKUCHI \& Yoshihiro MIZOGUCHI

Abstract collision systems simulated by cellular automata

MI2008-2 Eiji ONODERA

The intial value problem for a third-order dispersive flow into compact almost

Hermitian manifolds

MI2008-3 Hiroaki KIDO

On isosceles sets in the 4-dimensional Euclidean space

MI2008-4 Hirofumi NOTSU

Numerical computations of cavity flow problems by a pressure stabilized characteristiccurve finite element scheme

MI2008-5 Yoshiyasu OZEKI

Torsion points of abelian varieties with values in nfinite extensions over a padic field

MI2008-6 Yoshiyuki TOMIYAMA

Lifting Galois representations over arbitrary number fields

MI2008-7 Takehiro HIROTSU \& Setsuo TANIGUCHI

The random walk model revisited

MI2008-8 Silvia GANDY, Masaaki KANNO, Hirokazu ANAI \& Kazuhiro YOKOYAMA Optimizing a particular real root of a polynomial by a special cylindrical algebraic decomposition

MI2008-9 Kazufumi KIMOTO, Sho MATSUMOTO \& Masato WAKAYAMA

Alpha-determinant cyclic modules and Jacobi polynomials 
MI2008-10 Sangyeol LEE \& Hiroki MASUDA

Jarque-Bera Normality Test for the Driving Lévy Process of a Discretely Observed Univariate SDE

MI2008-11 Hiroyuki CHIHARA \& Eiji ONODERA

A third order dispersive flow for closed curves into almost Hermitian manifolds

MI2008-12 Takehiko KINOSHITA, Kouji HASHIMOTO and Mitsuhiro T. NAKAO

On the $L^{2}$ a priori error estimates to the finite element solution of elliptic problems with singular adjoint operator

MI2008-13 Jacques FARAUT and Masato WAKAYAMA

Hermitian symmetric spaces of tube type and multivariate Meixner-Pollaczek polynomials

MI2008-14 Takashi NAKAMURA

Riemann zeta-values, Euler polynomials and the best constant of Sobolev inequality

MI2008-15 Takashi NAKAMURA

Some topics related to Hurwitz-Lerch zeta functions

MI2009-1 Yasuhide FUKUMOTO

Global time evolution of viscous vortex rings 